\title{
Implicit and Explicit Spacecraft Gyro Calibration
}

\author{
Itzhack Y. Bar-Itzhack \\ Technion-Israel Institute of Technology, Haifa, Israel, 32000 \\ and \\ Richard R. Harman \\ NASA Goddard Space Flight Center, Greenbelt, MD, 20771
}

\begin{abstract}
This paper presents a comparison between two approaches to sensor calibration. According to one approach, called explicit, an estimator compares the sensor readings to reference readings, and uses the difference between the two to estimate the calibration parameters. According to the other approach, called implicit, the sensor error is integrated to form a different entity, which is then compared with a reference quantity of this entity, and the calibration parameters are inferred from the difference. In particular this paper presents the comparison between these approaches when applied to in-flight spacecraft gyro calibration. Reference spacecraft rate is needed for gyro calibration when using the explicit approach; however, such reference rates are not readily available for in-flight calibration. Therefore the calibration parameter-estimator is expanded to include the estimation of that reference rate, which is based on attitude measurements in the form of attitude-quaternion. A comparison between the two approaches is made using simulated data. It is concluded that the performances of the two approaches are basically comparable. Sensitivity tests indicate that the explicit filter results are essentially insensitive to variations in given spacecraft dynamics model parameters.
\end{abstract}

\section{Introduction}

TN-flight sensor calibration is a crucial element in spacecraft (SC) preparation for a successful mission and much work has been done on this subject, the latest being the work reported in Ref. 1. During the first stage of the calibration process the sensor errors sources, which are called calibration parameters, are estimated. During the second stage, errors caused by these calibration parameters are removed from the sensor readings.

There are two approaches to sensor calibration; namely, explicit and implicit approaches. In the explicit approach an estimator compares the sensor readings to reference readings, and it uses the difference between the two to estimate the calibration parameters. On the other hand, in the implicit approach the sensor error is integrated to form a different entity, which is then compared with a reference quantity of this entity, and the calibration parameters are inferred from the difference. If, for example, the sensors are accelerometers, then using the explicit approach the estimator compares the acceleration readings to reference acceleration. If the implicit approach is applied, then the accelerometer readings are properly integrated to generate velocity and the latter is compared with the reference velocity. The estimator uses this difference to estimate the calibration parameters. Similarly, if the sensors are gyroscopes, then in the explicit approach the estimator compares the measured rate with the reference one. In contrast, according to the implicit approach, the gyro readings are properly integrated to form attitude, which is then compared by the estimator to the reference attitude, and the estimator uses this difference to estimate the calibration parameters. As described, in both cases the estimator estimates the calibration parameters. Obviously, reference rate is required in the explicit approach to gyro calibration, and reference attitude is needed when the implicit approach is used. (It should be noted that by attitude we mean attitude in the wider sense; thus, two vector measurements constitute attitude reference in the wider sense because two vector measurements in the reference and body coordinates determine the attitude.)

When gyro calibration is performed on the ground it is easy to measure the reference rates to which the gyro outputs are compared. In-flight, however, the reference rate is not readily available; therefore, the rate has to be estimated. This adds an additional complication to in-flight gyro calibration when the explicit approach is used.

An example of the explicit approach can be found in Ref. 2. An improvement is presented in Ref. 3 where the calibration of the AQUA satellite gyroscopes was treated. Since the calibration was done in-flight, a reference angular

\footnotetext{
\# Sophie and William Shamban Professor of Aerospace Engineering, Faculty of Aerospace Engineering, ibaritz átechnion.ac.11, + 972-4-829-3196, Fellow

Aerospace Engineer, Flight Dynamics Analysis Branch, Code 595, richard.r.harman@nasa.gov, 301-286-5125.
} 
rate was not readily available. Therefore measured attitude was used to estimate the reference angular rate vector. Subsequently an estimator used the difference between the estimated reference rate and the gyro-measured rate to estimate the calibration parameters. Actually, the two estimators were combined into one Kalman filter. Because the rate estimation was based on Euler's equation that describes the SC angular dynamics, the rate estimation was nonlinear. Classically, an Extended Kalman filter is used to estimate the state vector of a system in which either the dynamics or the measurement model or both are nonlinear. However, it was found in several cases ${ }^{3-5}$ that when Euler's equations describing SC angular dynamics are used in the dynamics model the use of the Pseudo-Linear Kalman Filter (PSELIKA) ${ }^{6}$ produced very good estimates of the $\mathrm{SC}$ angular velocity.

A simple block diagram that explains the explicit approach to calibration is shown in Fig. 1. A more detailed description is presented in Fig. 2. The implicit approach is normally used for calibrating the inertial measuring unit in inertial navigation systems ${ }^{7}$ and is being used in spacecraft sensor calibration too (see e.g Ref. 8, 9). The methodology that governs the implicit approach when applied to gyro calibration is presented in a simple block diagram in Fig. 3 and a more elaborate description is shown in Fig. 4.

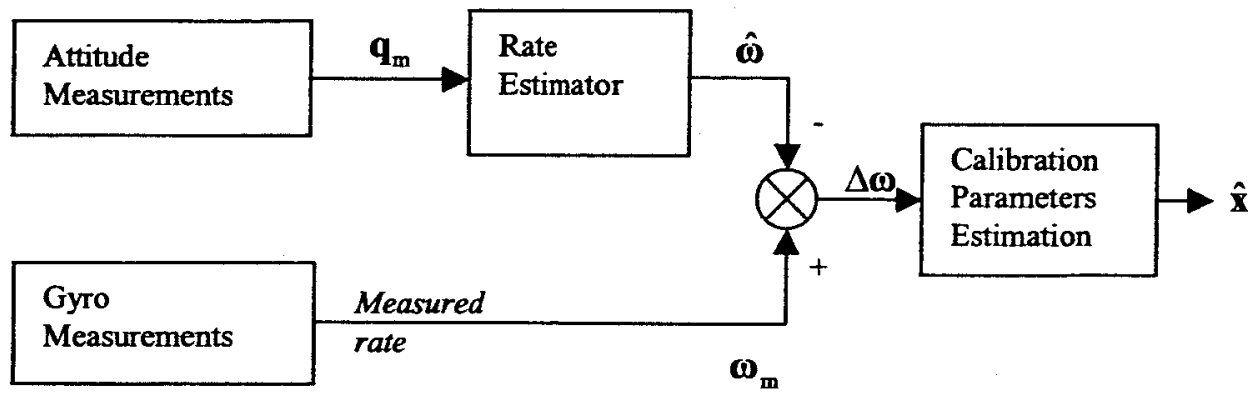

Fig. 1: Explicit Estimation of the Calibration Parameters.

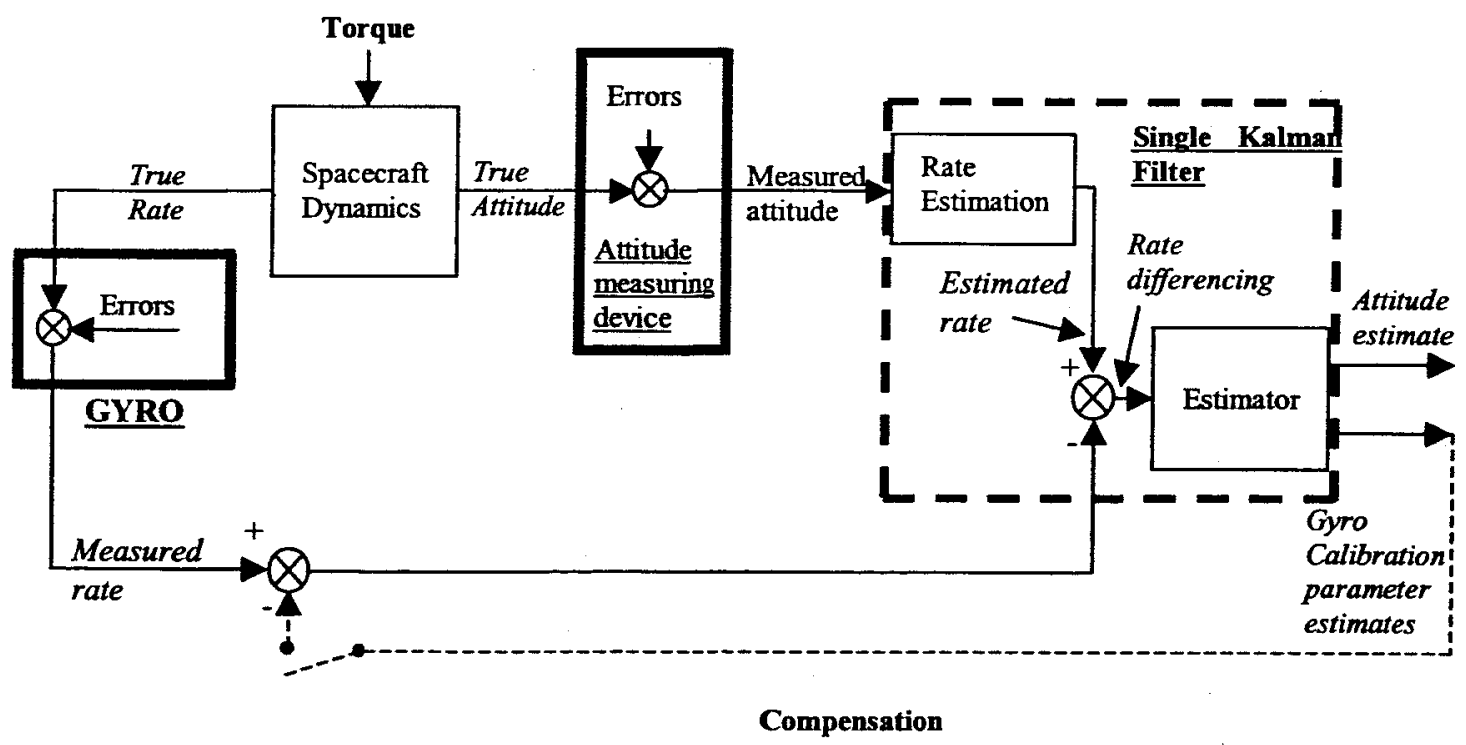

Fig. 2: A Detailed Description of the Explicit Estimation of the Calibration Parameters. 


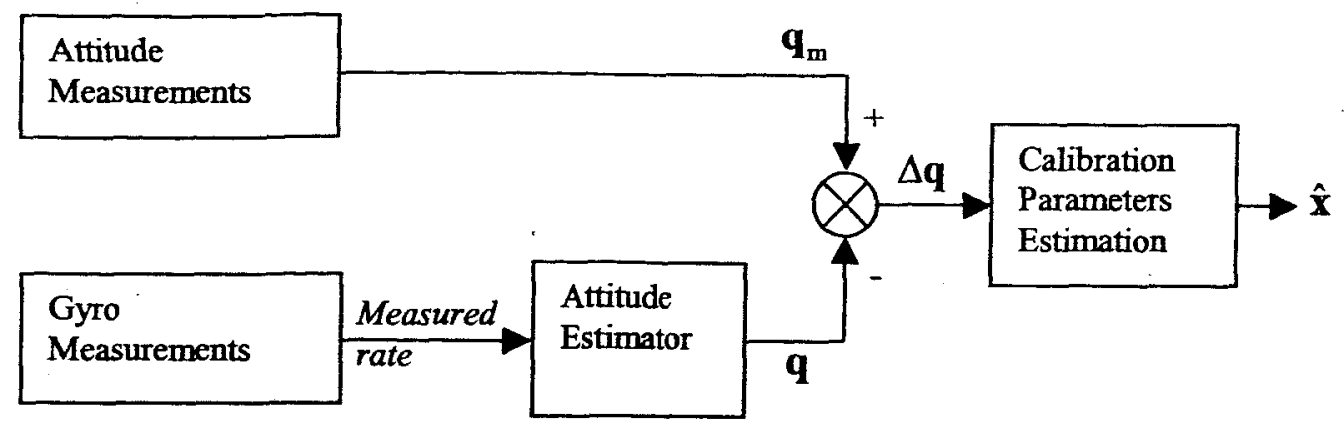

Fig. 3: Implicit Estimation of the Calibration Parameters.

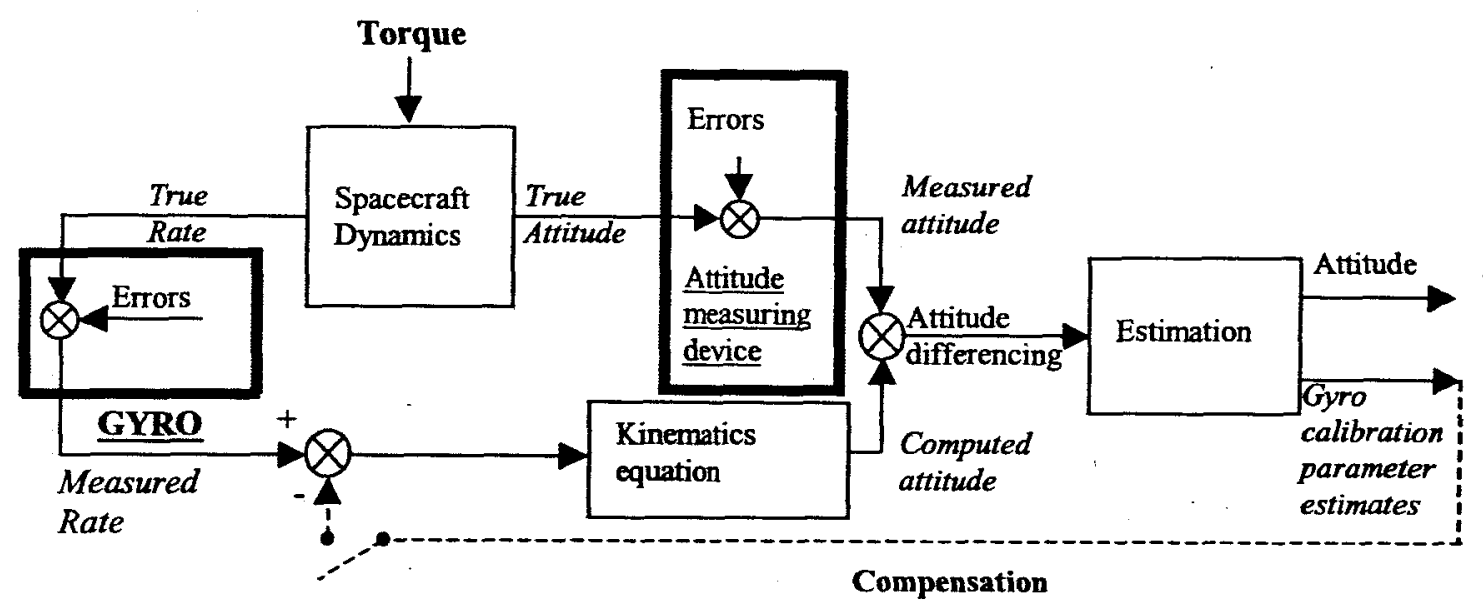

Fig. 4: A Detailed Description of the Implicit Estimation of the Calibration Parameters.

The purpose of this work is to compare between the explicit and the implicit approaches using simulated as well as real gyro data. In the next section we derive the gyro error model due to the calibration parameters. In Section III we develop the model employed by the explicit gyro-calibration estimator, and in the Section IV we present the model used by the implicit gyro - calibration estimator. Then, in section V, we show how the estimated calibration parameters are used to compensate the gyro readings. In Section VI we discuss some implementation issues, and show simulation results in Section VII. In the last section we draw our conclusions from this work.

\section{The Gyro Error Model}

The gyro errors that are considered in this work are: misalignment, scale factor error, and bias (constant drift rate). The gyro error model is a linear model, which associates small error sources to the gyro outputs. Due to the linearity of the model we can model the contribution of each error source independently and then sum up all the contributions into one linear model. We start the description of the error model by deriving the expression for the gyro misalignments.

\section{II.1 Misalignment Model}

The assumed direction of the sensitive axis of gyro $x$, which is one of the three considered gyro axes, is presented in Fig. 5 . The body coordinate axes are also presented and are denoted by $\mathbf{X}, \mathbf{Y}$, and $\mathbf{Z}$. The orientation of this gyro is expressed by a vector of unit length in the direction of the gyro sensitive (input) axis. It can be seen that 


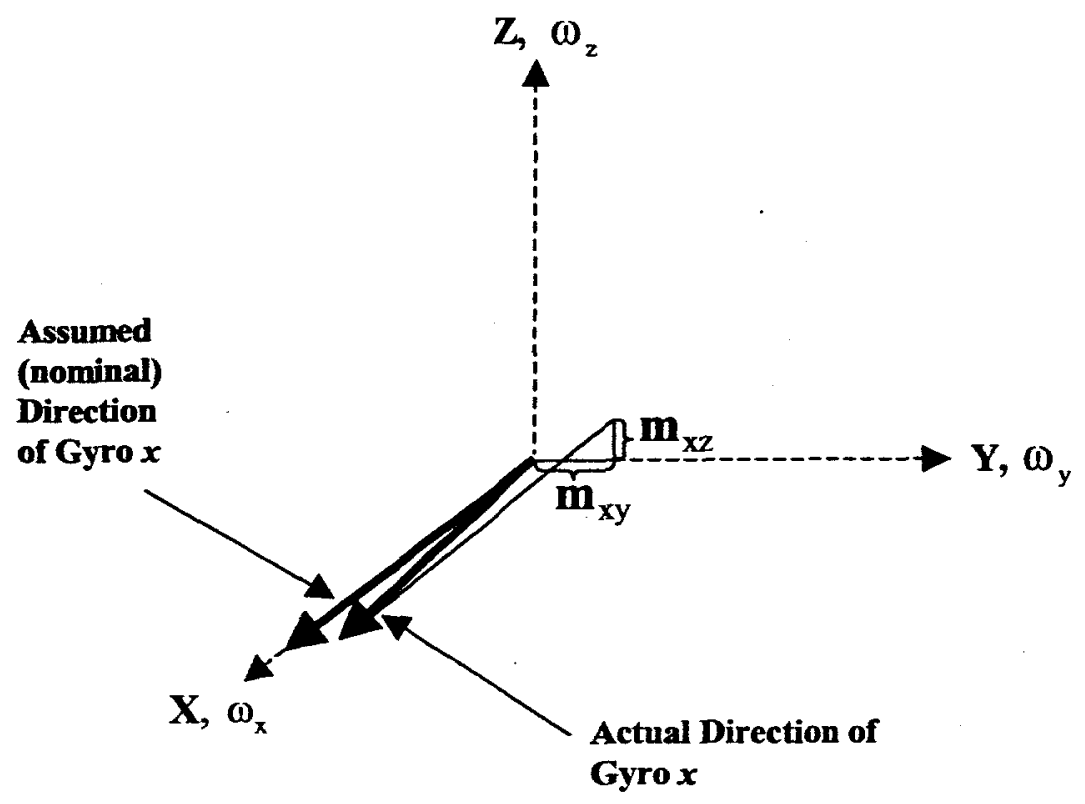

Fig. 5: Misalignment definition of the $x$-gyro sensitive axis.

the resulting rate error due to misalignment of all three gyros is:

$$
\Delta \boldsymbol{\omega}^{\mathrm{m}}=\left[\begin{array}{cccccc}
\omega_{y} & \omega_{z} & 0 & 0 & 0 & 0 \\
0 & 0 & \omega_{x} & \omega_{z} & 0 & 0 \\
0 & 0 & 0 & 0 & \omega_{x} & \omega_{y}
\end{array}\right]\left[\begin{array}{l}
m_{x y} \\
m_{x z} \\
m_{y x} \\
m_{y z} \\
m_{z x} \\
m_{z y}
\end{array}\right]
$$

where $\mathrm{m}_{\mathrm{ij}}$ is the projection of the $i$-gyro sensitive axis on the $j$ body-axis that is perpendicular to the $i$ body-axis. Since the sensitive axis is described by a unit vector and due to the proximity of the gyro sensitive axis to the respective body-axis, $\mathrm{m}_{\mathrm{ij}}$ is a misalignment angle, assumed to be small, expressed in radians. The elements $\omega_{x, y, z}$ are the angular velocity components measured by the gyros. Let,

$$
\boldsymbol{\Omega}^{\mathrm{m}}=\left[\begin{array}{cccccc}
\omega_{y} & \omega_{z} & 0 & 0 & 0 & 0 \\
0 & 0 & \omega_{\mathrm{x}} & \omega_{z} & 0 & 0 \\
0 & 0 & 0 & 0 & \omega_{\mathrm{x}} & \omega_{\mathrm{y}}
\end{array}\right]
$$

and

$$
\mathbf{m}^{\mathrm{T}}=\left[\begin{array}{llllll}
\mathrm{m}_{\mathrm{xy}} & \mathrm{m}_{\mathrm{xz}} & \mathrm{m}_{\mathrm{yx}} & \mathrm{m}_{\mathrm{yz}} & \mathrm{m}_{\mathrm{x}} & \mathbf{m}_{\mathrm{zy}}
\end{array}\right]
$$

Then Eq. (1) can be rewritten as, 


$$
\Delta \omega^{\mathbf{m}}=\Omega^{\mathbf{m}} \mathbf{m}
$$

\section{H.2 Scale Factor Error Model}

As mentioned, additional error sources that cause the difference between the correct value of the actual rates and their measurements are the scale factor errors. The error model for the scale factor errors is simply

$$
\Delta \omega^{k}=\left[\begin{array}{c}
\omega_{x} k_{x} \\
\omega_{y} k_{y} \\
\omega_{z} k_{z}
\end{array}\right]
$$

where the superscript $k$ denotes the fact that this error is caused by gyro scale factor errors, assumed to be small, and $k_{i}$ is the scale factor error of gyro $i, i=x, y, z$. Eq. (5) can be written as follows

$$
\Delta \omega^{k}=\left[\begin{array}{ccc}
\omega_{x} & 0 & 0 \\
0 & \omega_{y} & 0 \\
0 & 0 & \omega_{z}
\end{array}\right]\left[\begin{array}{l}
k_{x} \\
k_{y} \\
k_{z}
\end{array}\right]
$$

Define

$$
\Omega^{k}=\left[\begin{array}{ccc}
\omega_{x} & 0 & 0 \\
0 & \omega_{y} & 0 \\
0 & 0 & \omega_{z}
\end{array}\right]
$$

and

then Eq. (6) can be written as

$$
\mathbf{k}^{\mathrm{T}}=\left[\begin{array}{lll}
\mathbf{k}_{\mathrm{x}} & \mathbf{k}_{\mathrm{y}} & \mathbf{k}_{\mathrm{z}}
\end{array}\right]
$$

$$
\Delta \omega^{k}=\Omega^{k} \mathbf{k}
$$

\section{I.3 Bias Model}

The bias error model is quite simple and is given by

$$
\Delta \omega^{b}=I_{3} b
$$

where $I_{3}$ is the third order identity matrix and

$$
\mathbf{b}=\left[\begin{array}{l}
b_{x} \\
b_{y} \\
b_{z}
\end{array}\right]
$$

where $\mathrm{x}, \mathrm{y}, \mathrm{z}$ are the corresponding gyro axes.

\section{II.4 The Augmented Gyro Error Model}

The total gyro error is the sum of all the error discussed before; namely, misalignment, scale factor and bias errors; that is 


$$
\Delta \omega=\Delta \omega^{m}+\Delta \omega^{k}+\Delta \omega^{b}
$$

Using Eqs. (4), (7.c), and (8) we get

$$
\Delta \omega=\Omega^{\mathrm{m}} \mathbf{m}+\Omega^{\mathrm{k}} \mathbf{k}+\mathrm{I}_{\mathbf{3}} \mathbf{b}
$$

The last equation can be written in the following form

$$
\Delta \omega=\left[\begin{array}{lll}
\Omega^{m} & \Omega^{k} & I_{3}
\end{array}\right]\left[\begin{array}{l}
m \\
k \\
b
\end{array}\right]
$$

Define $H\left(\omega_{r}\right)\left(\omega_{r}\right.$ is the real angular rate vector) as follows

Also let

$$
H\left(\omega_{r}\right)=\left[\begin{array}{lll}
\Omega^{m} & \Omega^{k} & I_{3}
\end{array}\right]
$$

then Eq. (10.c) can be written as

$$
\mathbf{x}^{T}=\left[\begin{array}{lll}
\mathbf{m}^{\mathrm{T}} & \mathbf{k}^{\mathrm{T}} & \mathbf{b}^{\mathrm{T}}
\end{array}\right]
$$

$$
\Delta \omega=H\left(\omega_{r}\right) x
$$

\section{The Explicit Gyro Calibration Algorithm}

Attitude information and rates measured by the gyros are available in-flight but, unlike ground calibration, the reference rates needed for explicit calibration are not readily available. Therefore, as mentioned earlier, in this case we estimate the angular rate vector while estimating the calibration parameters. We use the attitude information to estimate the angular rate. The attitude information can be supplied in various ways; namely, it can be in the form of raw vector measurements or it can be given in an already processed form as attitude quaternion, for example. The estimation of the angular rate vector hinges on the following Euler's equation that describes the SC angular dynamics

$$
\dot{\omega}=I^{-1}[(I \omega+h) \times] \omega+I^{-1}(T-\dot{h})
$$

where $I$ is the $S C$ inertia tensor, $[(I \omega+\mathbf{h}) \times]$ is the cross product matrix of the vector $(I \omega+\mathbf{h})$ where a cross product of a general vector $\mathbf{a}$ is defined as follows

$$
[a \times]=\left[\begin{array}{ccc}
0 & -a_{z} & a_{y} \\
a_{z} & 0 & -a_{x} \\
-a_{y} & a_{x} & 0
\end{array}\right]
$$

$\mathbf{h}$ is the angular momentum of the momentum wheels, $\dot{h}=\mathrm{dh} / \mathrm{dt}$ and $\mathbf{T}$ is the external torque operating on the SC.

As mentioned before, the reference rate is estimated from the measured attitude. This necessitates the inclusion of the attitude kinematics in the estimator dynamics equation. Suppose that the attitude is measured by autonomous star trackers (AST) ${ }^{10}$ that yield the measured quaternion. The quaternion kinematics equation is expressed by ${ }^{11}$

$$
\dot{\mathbf{q}}=\frac{1}{2} \Omega \mathbf{q}
$$


where

$$
\Omega=\left[\begin{array}{cccc}
0 & \omega_{z} & -\omega_{y} & \omega_{x} \\
-\omega_{z} & 0 & \omega_{x} & \omega_{y} \\
\omega_{y} & -\omega_{x} & 0 & \omega_{z} \\
-\omega_{x} & -\omega_{y} & -\omega_{z} & 0
\end{array}\right]
$$

Equation (12.a) can be also written as

$$
\dot{\mathrm{q}}=\frac{1}{2} \mathrm{Q}_{\omega}
$$

where

$$
Q=\left[\begin{array}{ccc}
q_{1} & -q_{3} & q_{2} \\
q_{3} & q_{4} & -q_{1} \\
-q_{2} & q_{1} & q_{4} \\
-q_{1} & -q_{2} & -q_{3}
\end{array}\right]
$$

Because $\mathbf{X}$ is a constant vector, it obeys the following differential equation

$$
\dot{\mathbf{x}}=\mathbf{0}
$$

We can combine Eqs. (11), (12.c) and (13) into one dynamics equation, add white noise to the angular dynamics equation to account for model uncertainty, add a small quantity of white noise to the calibration parameter dynamics to better model them in case they are not really constant but rather vary slowly*, and add white noise to the quaternion kinematics equation to account for modeling inaccuracies. As a result we obtain the following dynamics equation

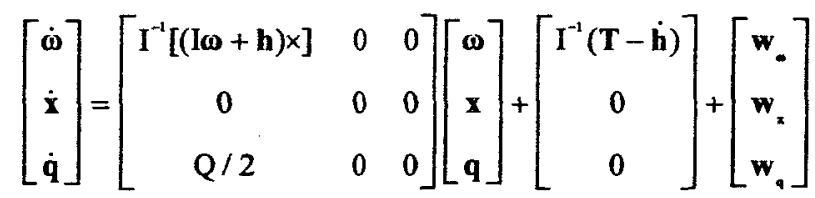

where $\mathbf{w}_{\omega}$ is the zero-mean white noise vector added to the angular dynamics, $\mathbf{w}_{\mathrm{x}}$ is the zero-mean white noise vector added to the calibration parameters, and $\mathbf{w}_{q}$ is the white noise added to the quaternion dynamics. Because the dynamics matrix is a function of $\boldsymbol{\omega}$ as well as $\mathbf{q}$, the dynamics equation presented in Eq. (14) is nonlinear, subsequently a nonlinear estimator is needed for estimating the state vector. The most appropriate estimator for the nonlinearity structure of this dynamics model is the PSELIKA algorithm ${ }^{3}$.

As explained before, we have two measurements to consider; namely, the gyro rate-measurements, $\omega_{m}$, and the quaternion measurement, $\mathbf{q}_{\mathbf{m}}$. The former consists of the true rate, the error due to the calibration parameters, $\mathbf{x}$, and some high frequency noise which is modeled as a zero-mean white noise, $\mathbf{v}_{\omega}$. Consequently we have

$$
\omega_{m}=\omega+\Delta \omega+v_{\omega}
$$

Using Eq. (10.f), the last equation can be written in the following matrix form.

\footnotetext{
* It is good practice to add some white noise to states in the filter model even when they are known to be constant (see Ref. 12).
} 


$$
\omega_{\mathrm{m}}=\left[\begin{array}{lll}
1 & H\left(\omega_{\mathrm{r}}\right) & 0
\end{array}\right]\left[\begin{array}{l}
\omega \\
\mathbf{x} \\
\mathbf{q}
\end{array}\right]+\mathbf{v}_{\mathrm{\omega}}
$$

The quaternion measurement is modeled by a combination of the true quaternion and a zero-mean white measurement noise vector, $\mathbf{v}_{\mathbf{q}}$.

$$
\mathbf{q}_{m}=\left[\begin{array}{lll}
0 & 0 & I
\end{array}\right]\left[\begin{array}{l}
\omega \\
\mathbf{x} \\
q
\end{array}\right]+\mathbf{v}_{q}
$$

As explained in the introduction, we combine the estimator that estimates the calibration parameters and that which estimates the rate vector into one estimator; therefore, we have to properly combine the two measurement equations. The merger of Eqs. (15.b) and (15.c) yields

$$
\left[\begin{array}{l}
\omega_{m} \\
q_{m}
\end{array}\right]=\left[\begin{array}{ccc}
C & H\left(\omega_{r}\right) & 0_{3 \times 4} \\
0_{4 \times 3} & 0_{4 \times 9} & I_{4 \times 4}
\end{array}\right]\left[\begin{array}{l}
\omega \\
x \\
q
\end{array}\right]+\left[\begin{array}{l}
v_{\omega} \\
v_{q}
\end{array}\right]
$$

Equation (14) and Eq. (15.d) constitute respectively the dynamics and the measurement equation for the augmented estimator used in the explicit approach to gyro calibration.

\section{The Implicit Gyro Calibration Algorithm}

As in the previous case, our goal now is to estimate $\mathbf{x}$, and, following the logic behind the implicit estimation approach, for that we need to know how $\mathbf{X}$ influences the attitude estimation. The true quaternion; that is, the quaternion that describes the true SC attitude, solves the differential equation (12.a) where $\Omega$ is a function of the true angular rate vector, $\omega$, which we do not know. We know and therefore we would rather use, the measured rate vector, $\omega_{m}$. According to Eq. (15.a), $\omega_{m}=\omega+\Delta \omega+v_{\omega}$ therefore

$$
\omega=\omega_{\mathbf{m}}-\Delta \omega-\mathbf{v}_{\omega}
$$

and correspondingly

$$
\Omega=\Omega_{\mathbf{m}}-\delta \Omega-\delta \Omega_{\mathrm{v}}
$$

where

$$
\Omega_{\mathrm{m}}=\left[\begin{array}{cccc}
0 & \omega_{z, \mathrm{~m}} & -\omega_{y, \mathrm{~m}} & \omega_{x, \mathrm{~m}} \\
-\omega_{x, \mathrm{~m}} & 0 & \omega_{x, \mathrm{~m}} & \omega_{y, \mathrm{~m}} \\
\omega_{y, \mathrm{~m}} & -\omega_{\mathrm{x}, \mathrm{m}} & 0 & \omega_{z, \mathrm{~m}} \\
-\omega_{x, \mathrm{~m}} & -\omega_{y, \mathrm{~m}} & -\omega_{z, \mathrm{~m}} & 0
\end{array}\right]
$$

and where $\delta \Omega$ and $\delta \Omega_{\mathrm{v}}$ are functions of $\omega$ and $\mathbf{v}_{\omega}$ respectively, and are in the format of Eq. (16.c). Using Eq. (16.b), Eq. (12.a) can be written as:

$$
\dot{\mathbf{q}}=\frac{1}{2} \Omega_{\mathrm{m}} \mathbf{q}-\frac{1}{2} \delta \Omega \mathbf{q}-\frac{1}{2} \delta \Omega_{\mathrm{v}} \mathbf{q}
$$

which can also be written as 


$$
\dot{\mathbf{q}}=\frac{1}{2} \Omega_{\mathrm{m}} \mathbf{q}-\frac{1}{2} \mathrm{Q} \Delta \omega-\frac{1}{2} \mathrm{Qv}
$$

where Q is given in Eq. (12.d). Using Eq. (10.f) in Eq. (17.b) yields

$$
\dot{\mathbf{q}}=\frac{1}{2} \Omega_{\mathbf{m}} \mathbf{q}-\frac{1}{2} \mathrm{QH}\left(\omega_{\mathrm{r}}\right) \mathbf{x}-\frac{1}{2} \mathrm{Qv} \text {. }
$$

Augmenting the last equation with Eq. (13) to which, as before, we add $\mathbf{w}_{x}$, yields

where

$$
\left[\begin{array}{c}
\dot{\mathbf{q}} \\
\dot{\mathbf{x}}
\end{array}\right]=\left[\begin{array}{cc}
\frac{1}{2} \Omega_{\mathbf{m}} & -\frac{1}{2} \mathrm{QH}\left(\omega_{\mathrm{r}}\right) \\
0 & 0
\end{array}\right]\left[\begin{array}{l}
\mathbf{q} \\
\mathbf{x}
\end{array}\right]+\left[\begin{array}{l}
\mathbf{w}_{\mathbf{q}} \\
\mathbf{w}_{\mathrm{x}}
\end{array}\right]
$$

$$
\mathbf{w}_{\mathrm{q}}=\frac{1}{2} \mathrm{Q} \mathbf{v}_{\text {. }}
$$

Eq. (17.d) is the dynamics model used by the estimator when we apply the implicit approach to gyro calibration. The measurement is the attitude measurement (and not the gyro measurements), which in our case is simply $\mathbf{q}_{m}$. From Eq. (15.c) it is obvious that here

$$
q_{m}=\left[\begin{array}{ll}
1 & 0
\end{array}\right]\left[\begin{array}{l}
\mathbf{q} \\
x
\end{array}\right]+v_{q}
$$

Equation (17.d) and Eq. (18) constitute, respectively, the dynamics and the measurement equation for the estimator used in the implicit approach to gyro calibration.

\section{Compensation}

To complete the calibration process we need to perform its second stage; namely, compensation of the gyro readings using the estimated calibration parameters. This stage is performed when either the explicit or the implicit approaches are used. It can be performed periodically during the estimation process or at its end. Compensation during the estimation process is preferable because then the models used by the filters, which are functions of the true angular velocity, become more accurate as the estimation proceeds. From Eq. (10.c) we obtain

$$
\Delta \hat{\boldsymbol{\omega}}=\left[\begin{array}{lll}
\Omega^{\mathbf{m}} & \Omega^{\mathrm{k}} & \mathrm{I}_{3}
\end{array}\right]\left[\begin{array}{c}
\hat{\mathbf{m}} \\
\hat{\mathbf{k}} \\
\hat{\mathbf{b}}
\end{array}\right]
$$

Since

$$
\omega=\omega_{\mathbf{m}}-\Delta \omega
$$

A calibrated gyro measurement, $\tilde{\boldsymbol{\omega}}$, is computed as follows

$$
\tilde{\boldsymbol{\omega}}=\boldsymbol{\omega}_{\mathrm{m}}-\Delta \hat{\boldsymbol{\omega}}
$$

Using Eq. (19.a) in the last equation yields the final gyro compensation formula 


$$
\tilde{\boldsymbol{\omega}}=\omega_{\mathrm{m}}-\left[\begin{array}{lll}
\Omega^{\mathrm{m}} & \Omega^{\mathrm{k}} & I_{3}
\end{array}\right]\left[\begin{array}{l}
\hat{\mathbf{m}} \\
\hat{\mathbf{k}} \\
\hat{\mathbf{b}}
\end{array}\right]
$$

\section{Implementation Considerations}

The dynamics matrix in Eq. (14) is a function of $\omega$ and $\mathbf{q}$ which are not available. Initially, however, we can evaluate the matrix using $\mathbf{q}_{\mathrm{m}}$ rather than $\mathbf{q}$ but when the attitude converges we can use the estimated quaternion, $\hat{\mathbf{q}}$, which is expected to be closer to $\mathbf{q}$. Similarly, initially we can use $\omega_{m}$, or the compensated gyro outputs (when compensation is done during the estimation process) rather than $\omega_{r}$ (which is not available to us) to evaluate the dynamics matrix of Eq. (14), the measurement matrix, $H$, of Eq. (15.d), and the dynamics matrix in Eq. (17.d). When the estimator converges in the explicit approach, it is better to switch to the estimated rate denoted by $\hat{\boldsymbol{\omega}}$, and when the estimate of $\mathbf{x}$ converges in the implicit approach, it is better to use the calibrated rate, $\tilde{\boldsymbol{\omega}}$, rather than $\boldsymbol{\omega}_{\mathbf{m}}$ because the calibrated gyro readings are normally expected to be closer to $\omega_{\mathrm{r}}$ more than $\omega_{\mathrm{m}}$ is.

\section{Test Results}

The algorithms were tested using simulated flight data. An angular rate profile was selected which assured complete observability of the estimated calibration parameters (see Fig. 6). It was assumed that no external torques operated on the SC. Thus, $\mathbf{T}$ was set to zero. In order to apply the correct wheel momentum that generated the desired $\omega$ profile, Eq. (11.a) was transformed into

$$
\dot{\mathbf{h}}=[-\boldsymbol{\omega} \times] \mathbf{h}+[(I \omega \times)] \omega-I \dot{\omega}+T
$$

and was solved for $\mathbf{h}$ where $\boldsymbol{\omega}$ and $\dot{\boldsymbol{\omega}}$ were, of course, known functions of time. The correct attitude quaternion was generated using the prescribed $\boldsymbol{\omega}$. The associated AST readings were simulated by adding the noise vector, $\mathbf{v}_{\mathrm{q}}$, to the measured quaternion (see Eq. (18)). That zero-mean noise was generated using a Gaussian random number generator with a standard deviation of $10^{-5}$. The following calibration parameters were used in the simulation.

$$
\begin{aligned}
& \mathbf{m}^{\mathrm{T}}=\left[\begin{array}{llllll}
0.1 & -0.1 & 0.1 & -0.1 & 0.1 & -0.1
\end{array}\right] \mathrm{deg} \\
& \mathbf{k}=\left[\begin{array}{c}
0.01 \\
0.01 \\
0.01
\end{array}\right] \quad(21 . \mathbf{b}) \quad \mathbf{b}=\left[\begin{array}{c}
+0.1 \\
-0.1 \\
+0.1
\end{array}\right] \mathrm{deg} / \mathrm{sec}
\end{aligned}
$$

The simulated gyro readings were generated using $\boldsymbol{\omega}$, the calibration parameters, and a random noise vector, $\mathbf{v}_{\boldsymbol{\omega}}$ (see Eq. (17.c). The latter vector was generated using a zero mean Gaussian random number generator with a standard deviation of $0.8 \mathrm{deg} / \mathrm{hr}$. The nominal inertia tensor was: 


$$
I=\left[\begin{array}{ccc}
100 & 0 & 0 \\
0 & 200 & 0 \\
0 & 0 & 300
\end{array}\right] \mathrm{kg} \cdot \mathrm{m}^{2}
$$

Each sensor provided data at a $1 \mathrm{~Hz}$ rate. The attitude profile started with an inertial period of 200 seconds (see Fig. 6) during which practically all of the gyro biases were estimated. This inertial period was followed by a 0.1 $\mathrm{deg} / \mathrm{sec}$ maneuver about the $x$-axis that lasted 200 seconds, followed by another 200 seconds of inertial period. Then at 600 seconds a $0.1 \mathrm{deg} / \mathrm{sec}$ maneuver about the $y$-axis was initiated which, too, lasted 200 seconds and was followed by 200 seconds of inertial period. At 1000 seconds a $0.1 \mathrm{deg} / \mathrm{sec}$ maneuver commenced, this time about the $z$-axis, lasting also 200 seconds. When the latter maneuver ended, at 1200 seconds, the SC stayed inertial until the end of the run at 1400 seconds. The two algorithms were applied to the AST readings and the algorithms produced estimates of the calibration parameters. In order to compare the performance of the two algorithms, figures of merit were computed for the misalignment, scale factor, and bias estimates based on the final values of the calibration estimates. The figures of merit were defined as follows:

$$
J_{m}=\sqrt{\sum_{i=1}^{6}\left(\frac{\varepsilon_{m, i}}{m_{i}}\right)^{2}} \quad \text { (21.a) } \quad J_{k}=\sqrt{\sum_{i=1}^{3}\left(\frac{\varepsilon_{k, i}}{k_{i}}\right)^{2}} \quad \text { (21.b) } \quad J_{b}=\sqrt{\sum_{i=1}^{3}\left(\frac{\varepsilon_{b, i}}{b_{i}}\right)^{2}}
$$

where:

$$
\begin{aligned}
& \varepsilon_{\mathrm{m}, \mathrm{i}}=\mathrm{i}^{\text {th }} \text { misalignment final estimation error. } \\
& \mathrm{m}_{\mathrm{i}}=\mathrm{i}^{\text {th }} \text { misalignment. } \\
& \varepsilon_{\mathrm{k}, \mathrm{i}}=\mathrm{i}^{\text {th }} \text { scale factor final estimation error. } \\
& \mathrm{k}_{\mathrm{i}}=\mathrm{i}^{\text {th }} \text { scale factor. } \\
& \varepsilon_{\mathrm{b}, \mathrm{i}}=\mathrm{i}^{\text {th }} \text { bias final estimation error. } \\
& \mathrm{b}_{\mathrm{i}}=\mathrm{i}^{\text {th }} \text { bias. }
\end{aligned}
$$

\section{VII.1 Results with the Explicit Filter}

Figure 6 presents the true angular rates during the simulation (in black) and the estimates (in red). We note that good rate estimates are imperative for obtaining good calibration parameter estimates when using the explicit approach. The true and estimated values of the gyro misalignments are presented in figure 7 . The scale factors are compared in figure 8 , and figure 9 presents the same for the gyro bias values.

\section{VII.2 Results with the Implicit Filter}

The true and estimated values of the gyro misalignments are presented in figure 10. The scale factors are compared in figure 11 , and figure 12 presents the same for the gyro bias values. 

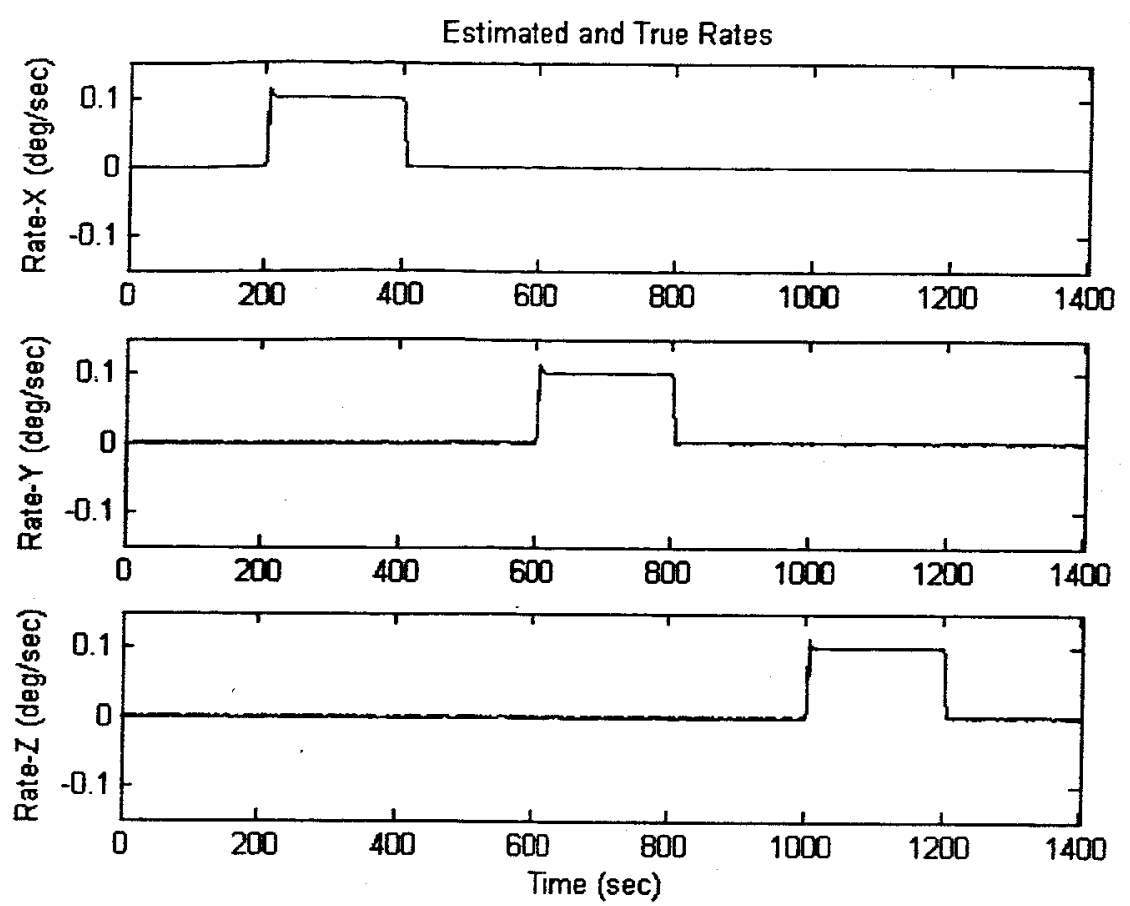

Fig. 6: True (broken black line) and estimated (red line) angular rate components.
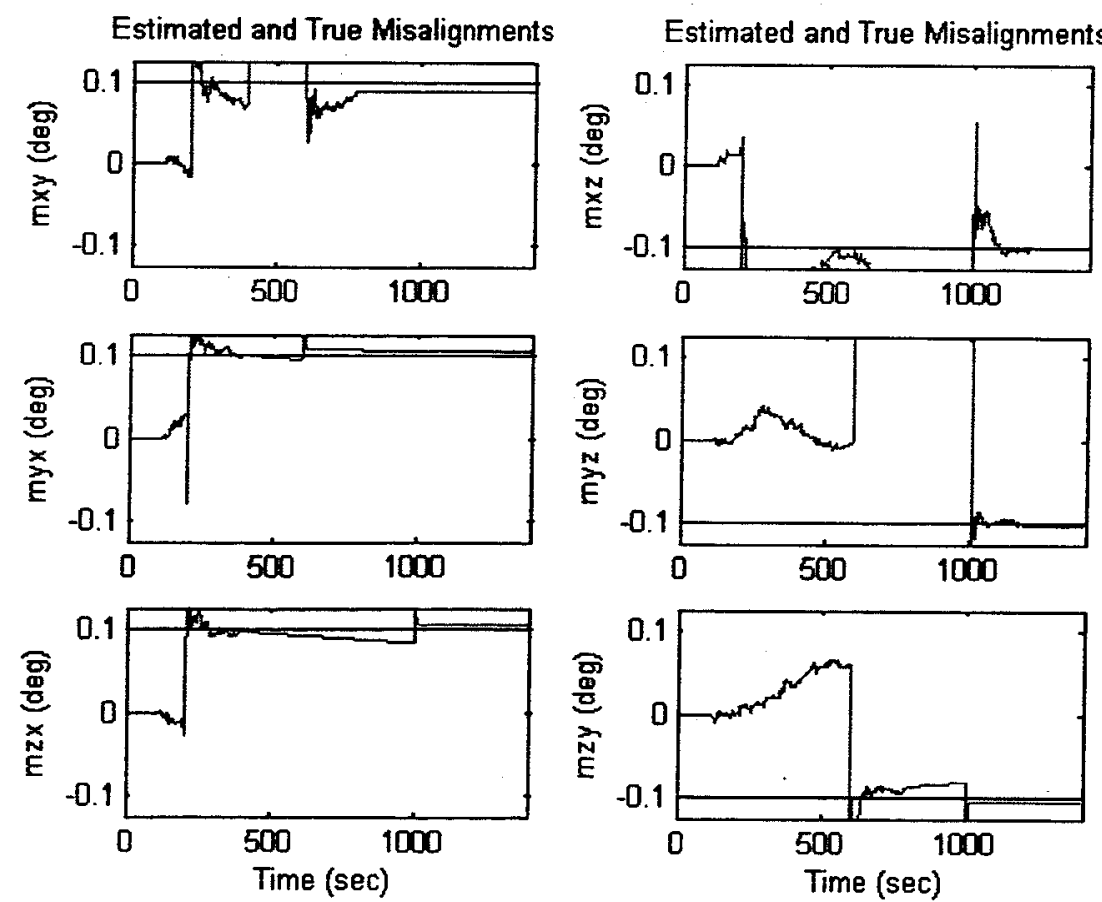

Fig. 7: True (black line) and explicit estimate (red line) gyro misalignments. 

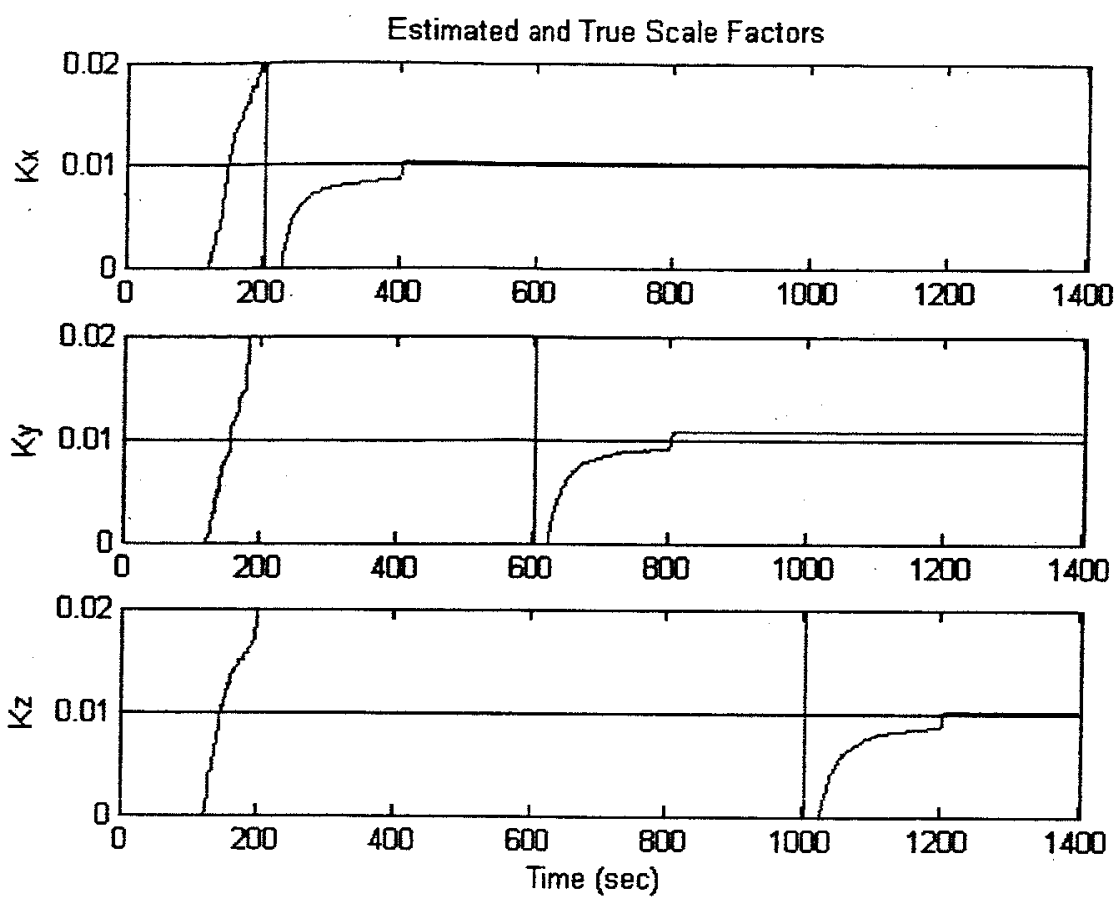

Fig. 8: True (black line) and explicit estimate (red line) gyro scale factors.
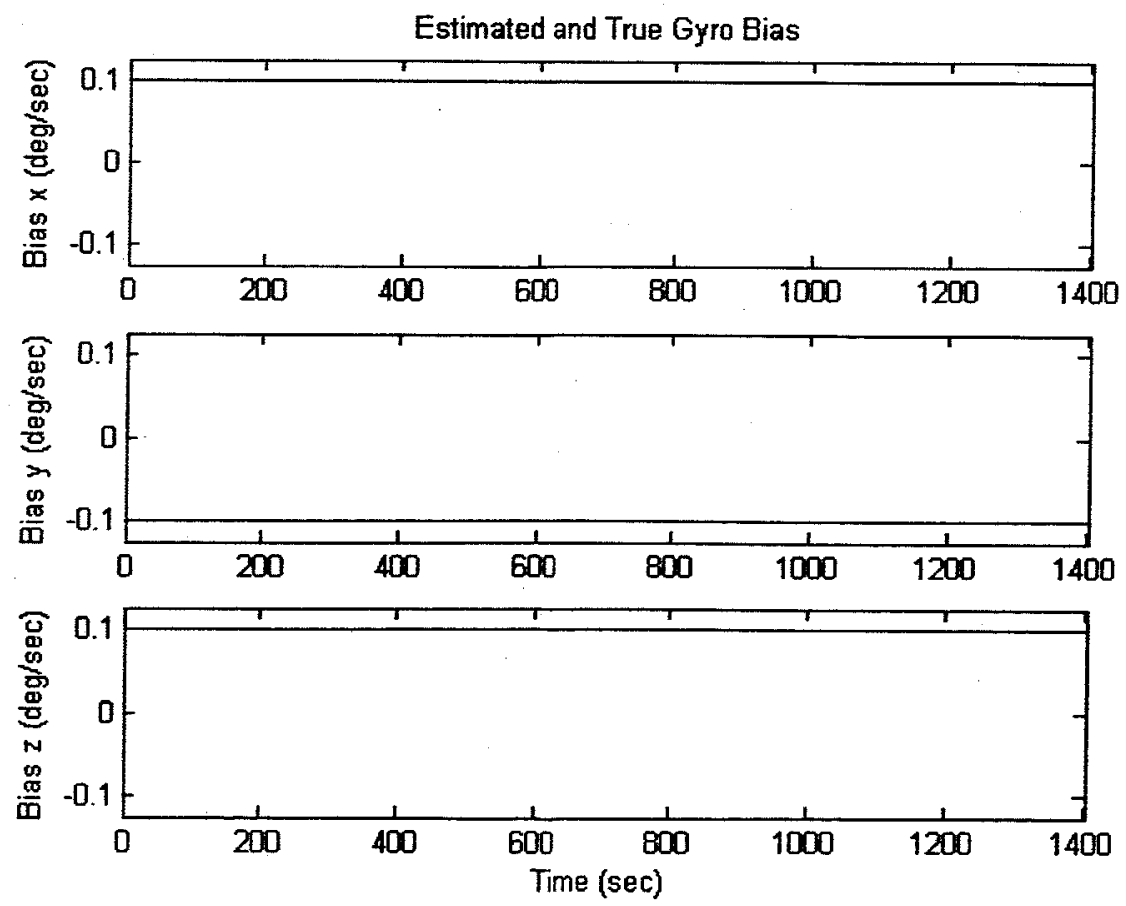

Fig. 9: True (black line) and explicit estimate (red line) of gyro biases. 

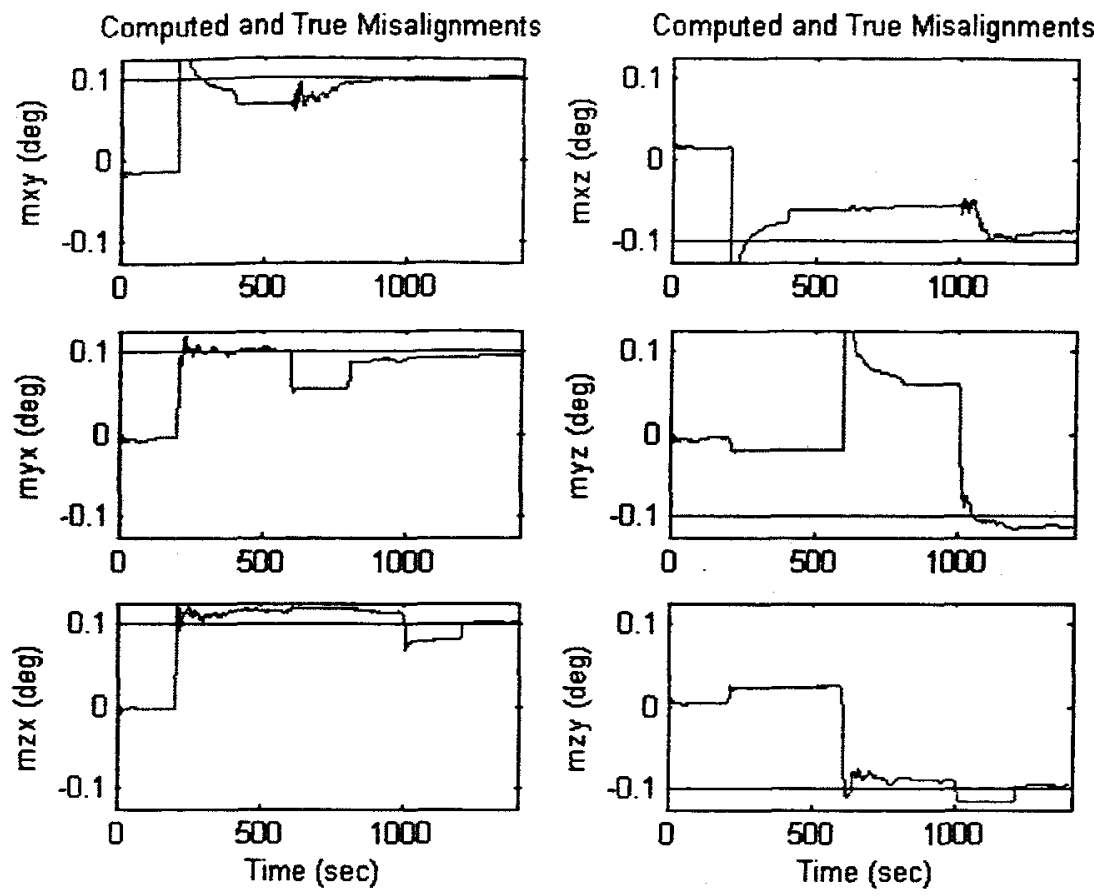

Fig. 10: True (black line) and implicit estimate (red line) gyro misalignments.
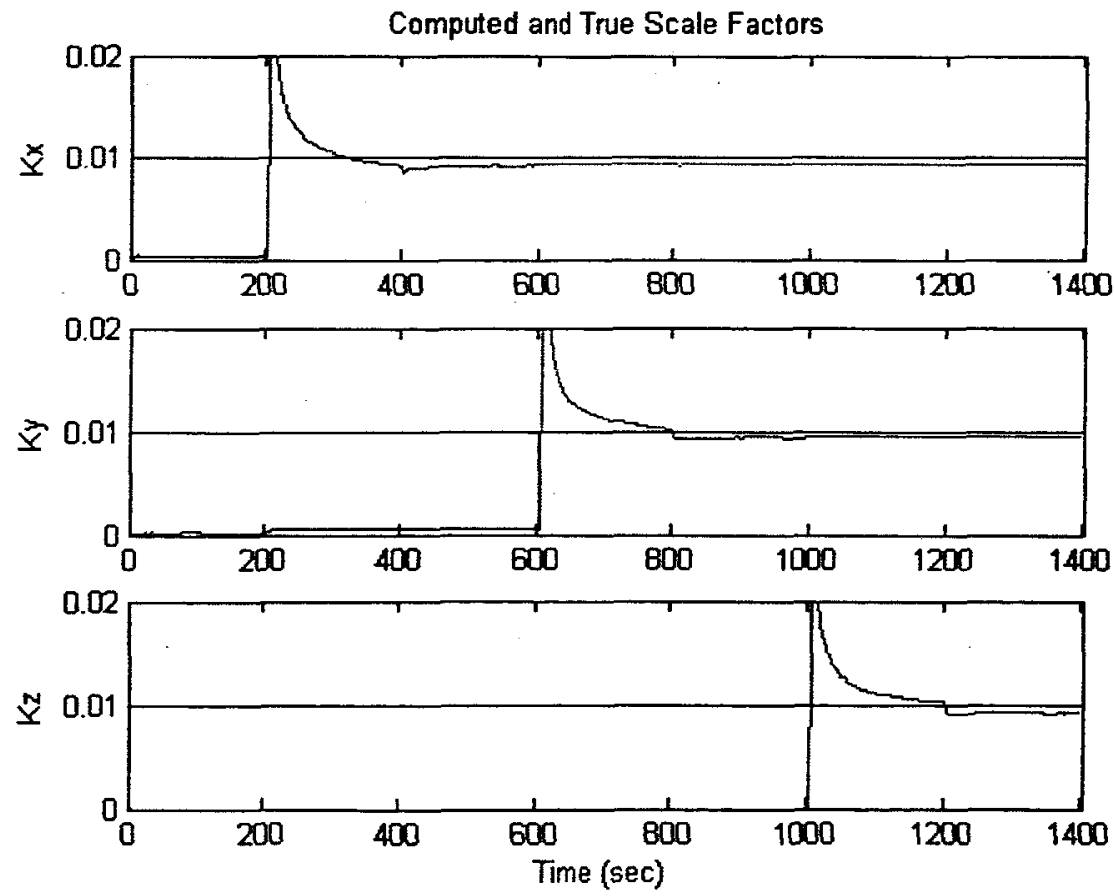

Fig. 11: True (black line) and implicit estimate (red line) gyro scale factors. 

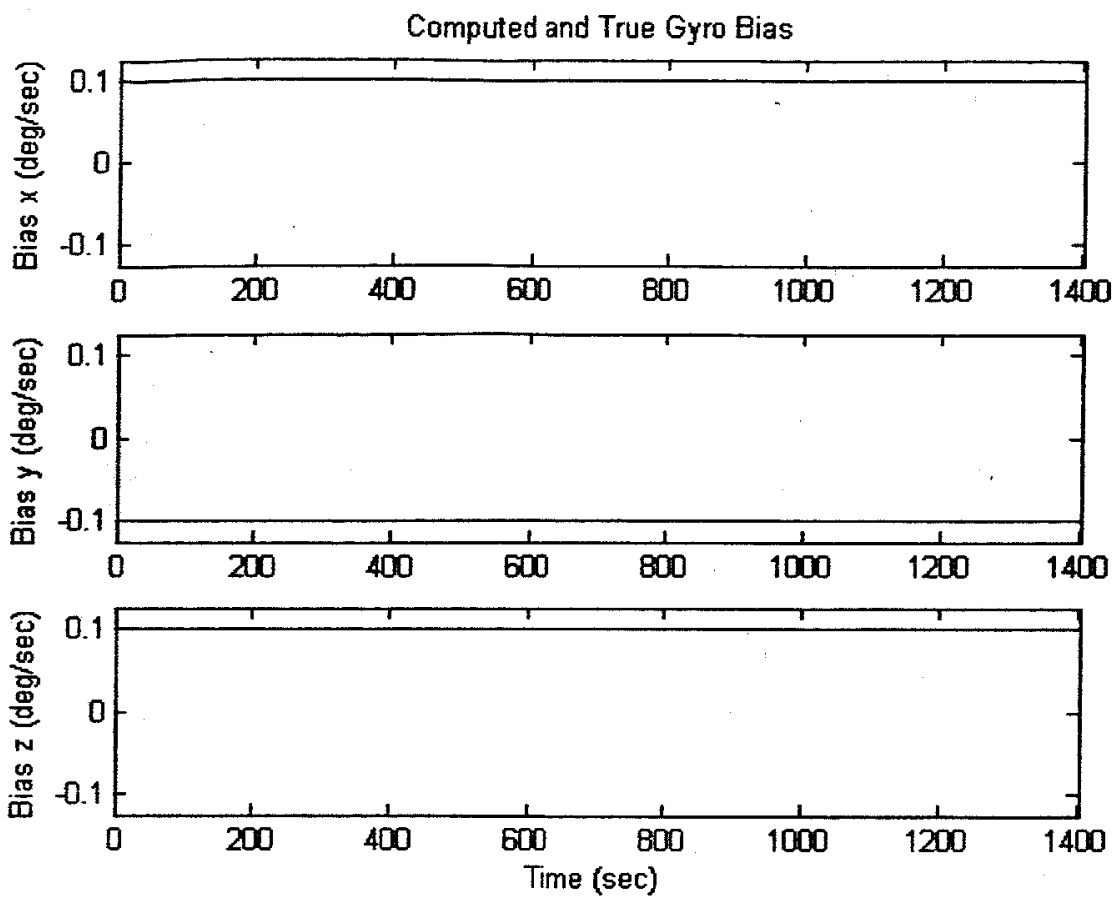

Fig. 12: True (black line) and implicit estimate (red line) gyro biases.

\section{VII.2 Comparison of the Figures of Merit}

The three pairs of figures of merit, one for the misalignment, one for the scale factor and one for the bias estimation errors are presented in Table $I$. It is seen that while the explicit algorithm estimates the misalignments and scale factors better than the implicit algorithm, the latter does better in estimating the biases. However the changes are minor and depend heavily on our ability to tune each filter. In the scenario presented, the performance of the two approaches is comparable.

Table I: Figures of Merit

\begin{tabular}{|l|c|c|}
\cline { 2 - 3 } \multicolumn{1}{c|}{} & Explicit & Implicit \\
\hline $\mathrm{J}_{\mathrm{m}}$ (for misalignment) & 0.14987098 & 0.19447241 \\
\hline $\mathrm{J}_{\mathrm{k}}$ (for scale factor) & 0.07091744 & 0.01163662 \\
\hline $\mathrm{J}_{\mathrm{b}}$ (for bias) & 0.00033426 & 0.00028284 \\
\hline
\end{tabular}

\section{VII.3 Sensitivity Tests}

Unlike the implicit filter, the explicit filter uses a model of the SC dynamics in terms of Euler's equation. In the preceding simulations of the explicit filter we used in the filter-model the SC parameters that were also used in the simulation of the angular rate profile (we designate this case as the nominal case). It is important to examine the sensitivity of the filter results to uncertainty in these parameters. Table I presents the comparison between the nominal explicit nominal filter and the implicit filter. In Table II we present a comparison between the results obtained when using the nominal explicit filter and those obtained when the inertia matrix in the filter is $90 \%$ of its nominal value. In table III we present a comparison between the nominal case and the results obtained using the 
explicit filter where $I_{x x}$ in the filter is $10 \%$ higher than its nominal value, $I_{y y}$ is $10 \%$ lower, and $I_{z z}$ is higher than its nominal value. Finally, in Table III we present the comparison when the components of the filter $\mathbf{h}$ are $10 \%$ lower than their nominal values. It is obvious that the filter is not sensitive to parameter uncertainty.

Table II: Figures of Merit of the Nominal Case and the Case of $10 \%$ Reduction in the Inertia

\begin{tabular}{|l|c|c|}
\cline { 2 - 3 } \multicolumn{1}{c|}{} & Nominal & Mismatched \\
\hline $\mathrm{J}_{\mathrm{m}}$ (for misalignment) & 0.14987098 & 0.14968341 \\
\hline $\mathrm{J}_{\mathrm{k}}$ (for scale factor) & 0.07091744 & 0.07080843 \\
\hline $\mathrm{J}_{\mathrm{b}}$ (for bias) & 0.00033426 & 0.00033426 \\
\hline
\end{tabular}

Table III: Figures of Merit of the Nominal Case and the Case of Alternate Inertia Variation

\begin{tabular}{|l|c|c|}
\cline { 2 - 3 } \multicolumn{1}{c|}{} & Nominal & Mismatched \\
\hline $\mathrm{J}_{\mathrm{m}}$ (for misalignment) & 0.14987098 & 0.15031518 \\
\hline $\mathrm{J}_{\mathrm{k}}$ (for scale factor) & 0.07091744 & 0.07087014 \\
\hline $\mathrm{J}_{\mathrm{b}}$ (for bias) & $\mathbf{0 . 0 0 0 3 3 4 2 6}$ & 0.00033426 \\
\hline
\end{tabular}

Table IV: Figures of Merit of the Nominal Case and the Case of $10 \%$ Reduction in $h$.

\begin{tabular}{|l|c|c|}
\cline { 2 - 3 } \multicolumn{1}{c|}{} & Nominal & Mismatched \\
\hline $\mathrm{J}_{\mathrm{m}}$ (for misalignment) & 0.14987098 & 0.14989170 \\
\hline $\mathrm{J}_{\mathrm{k}}$ (for scale factor) & 0.07091744 & 0.07091747 \\
\hline $\mathrm{J}_{\mathrm{b}}$ (for bias) & 0.00033426 & 0.00033426 \\
\hline
\end{tabular}

\section{Conclusions}

This paper presented a comparison between two approaches to the problem of SC sensor calibration. In particular, the problem of gyro misalignment, scale factor, and bias estimation was considered. The first approach, the explicit approach, utilizes attitude measurements to obtain rate estimates, which are compared to the gyromeasured rates in order to estimate the calibration parameters. The rate estimation and the calibration parameter estimation are performed by one pseudo-linear Kalman filter. The second approach, the implicit approach, uses the un-calibrated gyro outputs to compute an attitude that is compared with the measured attitude. The difference, which is a function of the calibration parameters, is also used in a pseudo-linear filter. In this paper the error models used by the filters of the two approaches were developed and excessive simulation runs were made for different values of calibration parameters. It was found that the performances of the two approaches were comparable. However, the results are sensitive to filter tuning. Sensitivity tests were carried out on the explicit filter dynamics model uncertainty. It was found that the explicit filter results were practically insensitive to inertia and wheel momentum uncertainties. In the present simulations quaternion observations were used, which were based on star tracker measurements. It is recommended that sun sensor and magnetometer measurements, which provide less accurate attitude determination, also be tested. 


\section{References}

${ }^{1}$ Thienel, J.K., Nonlinear Observer/Controller Designs for Spacecraft Attitude Control Systems With Uncalibrated Gyros," Ph. D. Dissertation, Department of Aerospace Engineering, University of Maryland, May 2004.

${ }^{2}$ Lambertson, M., Keat,J., and Scheidker, E., "Multimission Three-Axis Stabilized Spacecraft (MTASS) Flight Dynamics Support System (FDSS) Mathematical Background," NASA Goddard Space Flight Center, Publ.553FDD-93/032R0UD0, Sept.1993, pp. 3.3.2-1-3.3.2-10.

${ }^{3}$ Bar-Itzhack, I.Y., and Harman, R.R., "In-Space Calibration of a Skewed Gyro Quadruplet," ALAA J. of Guidance, Control, and Dynamics, Vol. 25, No. 5, Sept.-Oct. 2002, pp. 852-859.

${ }^{4}$ Bar-Itzhack, I.Y., Harman, R.R., and Choukroun, D., "State-Dependent Pseudo-Linear Filters for Spacecraft Attitude and Rate Estimation", AIAA Guidance, Navigation and Control Conference, Monterey, CA, USA, Aug. 58, 2002.

${ }^{5}$ Bar-Itzhack, I.Y., and Harman, R.R., "The MAP Spacecraft Angular State Estimation After Sensor Failure", AIAA Guidance, Navigation and Control Conference, Austin, TX, USA, Aug. 11-14, 2003.

${ }^{6}$ Bar-Itzhack, I.Y., and Harman, R.R., "Pseudolinear and State-Dependent Riccati Equation Filters for Angular Rate Estimation", ALAA J. of Guidance, Control, and Dynamics, Vol. 22, No. 5, Sept.-Oct. 1999, pp. 723-725.

${ }^{7}$ Chatfield, A.B., Fundamentals of High Accuracy Inertial Navigation, Vol. 174 Progress in Astronautics and Aeronautics, AIAA, 1997, pp. 93-106.

${ }^{8}$ Shuster, M.D., "Inflight Estimation of Spacecraft Sensor Alignment," $13^{\text {th }}$ Annual AAS Guidance and Control Conference, American Astronautical Society, Springfield, VA, 1990, pp. 253-274.

${ }^{9}$ Pittelkau, M.E., "Kalman Filtering for Spacecraft System Alignment Calibration," ALAA J. of Guidance, Control, and Dynamics, Vol. 24, No. 6, Nov.-Dec. 2001, pp. 1187-1195.

${ }^{10}$ Bezooijen, R.W.H., "AST Capabilities," Lockheed Martin Advanced Technology Center, Palo Alto, CA 95304-1191. (Slide presentation).

${ }^{11}$ Wertz J.R., (Ed.), Spacecraft Attitude Dynamics and Control, Reidel Publishing Co., Dordrecht, Holland, 1978, p. 512.

${ }^{12}$ Bryson, A.E. "Kalman Filter Divergence and Aircraft Motion Estimator", J. of Guidance and Control, Vol. 1, No. 1, January-February 1978, pp. 71-79. 